\title{
Some Delay Integral Inequalities on Time Scales and Their Applications in the Theory of Dynamic Equations
}

\author{
Qinghua Feng, ${ }^{1,2}$ Fanwei Meng, ${ }^{2}$ Yaoming Zhang, ${ }^{1}$ \\ Jinchuan Zhou, ${ }^{1}$ and Bin Zheng' \\ ${ }^{1}$ School of Science, Shandong University of Technology, Shandong, \\ Zibo 255049, China \\ 2 School of Mathematical Sciences, Qufu Normal University, Shandong, \\ Qufu 273165, China
}

Correspondence should be addressed to Qinghua Feng, fqhua@sina.com

Received 29 July 2011; Accepted 1 December 2011

Academic Editor: Agacik Zafer

Copyright (C) 2012 Qinghua Feng et al. This is an open access article distributed under the Creative Commons Attribution License, which permits unrestricted use, distribution, and reproduction in any medium, provided the original work is properly cited.

\begin{abstract}
We establish some delay integral inequalities on time scales, which on one hand provide a handy tool in the study of qualitative as well as quantitative properties of solutions of certain delay dynamic equations on time scales and on the other hand unify some known continuous and discrete results in the literature.
\end{abstract}

\section{Introduction}

During the past decades, with the development of the theory of differential and integral equations as well as difference equations, a lot of integral and difference inequalities have been discovered (e.g., see [1-13] and the references therein), which play an important role in the research of boundedness, global existence, stability of solutions of differential and integral equations as well as difference equations. On the other hand, Hilger [14] initiated the theory of time scales as a theory capable to contain both difference and differential calculus in a consistent way. Since then many authors have expounded on various aspects of the theory of dynamic equations on time scales including various inequalities on time scales (e.g., see [15-24], and the references therein). However, delay integral inequalities on time scales have been paid little attention so far. Recent results in this direction include the works of Li [25] and Ma and Pečarić [26] to our best knowledge. 
In this paper, we will establish some new delay integral inequalities on time scales, which unify some known continuous and discrete results in the literature. New explicit bounds for unknown functions concerned are obtained due to the presented inequalities. Some applications will be presented for the established results.

Throughout this paper, $\mathbb{R}$ denotes the set of real numbers and $\mathbb{R}_{+}=[0, \infty)$, while $\mathbb{Z}$ denotes the set of integers. For two given sets $G, H$, we denote the set of maps from $G$ to $H$ by $(G, H)$.

\section{Some Preliminaries on Time Scales}

A time scale is an arbitrary nonempty closed subset of the real numbers. In this paper, $\mathbb{T}$ denotes an arbitrary time scale. On $\mathbb{T}$ we define the forward and backward jump operators $\sigma \in(\mathbb{T}, \mathbb{T})$ and $\rho \in(\mathbb{T}, \mathbb{T})$ such that $\sigma(t)=\inf \{s \in \mathbb{T}, s>t\}, \rho(t)=\sup \{s \in \mathbb{T}, s<t\}$.

Definition 2.1. The graininess $\mu \in\left(\mathbb{T}, \mathbb{R}_{+}\right)$is defined by $\mu(t)=\sigma(t)-t$.

Remark 2.2. Obviously, $\mu(t)=0$ if $\mathbb{T}=\mathbb{R}$, while $\mu(t)=1$ if $\mathbb{T}=\mathbb{Z}$.

Definition 2.3. A point $t \in \mathbb{T}$ is said to be left-dense if $\rho(t)=t$ and $t \neq$ inf $\mathbb{T}$, right-dense if $\sigma(t)=t$ and $t \neq \sup \mathbb{T}$, left-scattered if $\rho(t)<t$, and right-scattered if $\sigma(t)>t$.

Definition 2.4. The set $\mathbb{T}^{\mathcal{k}}$ is defined to be $\mathbb{T}$ if $\mathbb{T}$ does not have a left-scattered maximum, otherwise it is $\mathbb{T}$ without the left-scattered maximum.

Definition 2.5. A function $f \in(\mathbb{T}, \mathbb{R})$ is called rd-continuous if it is continuous in right-dense points and if the left-sided limits exist in left-dense points, while $f$ is called regressive if $1+\mu(t) f(t) \neq 0 . C_{\text {rd }}$ denotes the set of rd-continuous functions, while $\mathfrak{R}$ denotes the set of all regressive and rd-continuous functions, and $\mathfrak{R}^{+}=\{f \mid f \in \mathfrak{R}, 1+\mu(t) f(t)>0$, for all $t \in \mathbb{T}\}$.

Definition 2.6. For some $t \in \mathbb{T}^{\kappa}$, and a function $f \in(\mathbb{T}, \mathbb{R})$, the delta derivative of $f$ at $t$ is denoted by $f^{\Delta}(t)$ (provided it exists) with the property such that for every $\varepsilon>0$ there exists a neighborhood $\mathfrak{U}$ of $t$ satisfying

$$
\left|f(\sigma(t))-f(s)-f^{\Delta}(t)(\sigma(t)-s)\right| \leq \varepsilon|\sigma(t)-s| \quad \forall s \in \mathfrak{U}
$$

Remark 2.7. If $\mathbb{T}=\mathbb{R}$, then $f^{\Delta}(t)$ becomes the usual derivative $f^{\prime}(t)$, while $f^{\Delta}(t)=f(t+1)-f(t)$ if $\mathbb{T}=\mathbb{Z}$, which represents the forward difference.

Definition 2.8. For $a, b \in \mathbb{T}$ and a function $f \in(\mathbb{T}, \mathbb{R})$, the Cauchy integral of $f$ is defined by

$$
\int_{a}^{b} f(t) \Delta t=F(b)-F(a)
$$

where $F^{\Delta}(t)=f(t), t \in \mathbb{T}^{\kappa}$.

The following two theorems include some important properties for delta derivative and the Cauchy integral on time scales. 
Theorem 2.9 (see [27]). If $f, g \in(\mathbb{T}, \mathbb{R})$, and $t \in \mathbb{T}^{\mathcal{k}}$, then

(i)

$$
f^{\Delta}(t)=\left\{\begin{array}{lll}
\frac{f(\sigma(t))-f(t)}{\mu(t)} & \text { if } \mu(t) \neq 0, \\
\lim _{s \rightarrow t} \frac{f(t)-f(s)}{t-s} & \text { if } \mu(t)=0 .
\end{array}\right.
$$

(ii) If $f, g$ are delta differentials at $t$, then $f g$ is also delta differential at $t$, and

$$
(f g)^{\Delta}(t)=f^{\Delta}(t) g(t)+f(\sigma(t)) g^{\Delta}(t)
$$

Theorem 2.10 (see [27]). If $a, b, c \in \mathbb{T}, \alpha \in \mathbb{R}$, and $f, g \in C_{\mathrm{rd}}$, then

(i) $\int_{a}^{b}[f(t)+g(t)] \Delta t=\int_{a}^{b} f(t) \Delta t+\int_{a}^{b} g(t) \Delta t$,

(ii) $\int_{a}^{b}(\alpha f)(t) \Delta t=\alpha \int_{a}^{b} f(t) \Delta t$,

(iii) $\int_{a}^{b} f(t) \Delta t=-\int_{b}^{a} f(t) \Delta t$,

(iv) $\int_{a}^{b} f(t) \Delta t=\int_{a}^{c} f(t) \Delta t+\int_{c}^{b} f(t) \Delta t$,

(v) $\int_{a}^{a} f(t) \Delta t=0$,

(vi) if $f(t) \geq 0$ for all $a \leq t \leq b$, then $\int_{a}^{b} f(t) \Delta t \geq 0$.

Definition 2.11. The cylinder transformation $\xi_{h}: \mathbb{C}_{h} \rightarrow \mathbb{Z}_{h}$ is defined by

$$
\xi_{h}(z)= \begin{cases}\frac{\log (1+h z)}{h}, & \text { if } h \neq 0\left(\text { for } z \neq-\frac{1}{h}\right) \\ z, & \text { if } h=0\end{cases}
$$

where Log is the principal logarithm function.

Definition 2.12. For $p \in \mathfrak{R}$ and $s, t \in \mathbb{T}$, the exponential function is defined by

$$
e_{p}(t, s)=\exp \left(\int_{s}^{t} \xi_{\mu(\tau)}(p(\tau)) \Delta \tau\right)
$$

Remark 2.13. If $\mathbb{T}=\mathbb{R}$, then for $s, t \in \mathbb{R}, e_{p}(t, s)=\exp \left(\int_{s}^{t} p(\tau) d \tau\right)$. If $\mathbb{T}=\mathbb{Z}$, then for $s, t \in \mathbb{Z}$ and $s<t, e_{p}(t, s)=\prod_{\tau=s}^{t-1}[1+p(\tau)]$. function.

The following two theorems include some known properties on the exponential

Theorem 2.14 (see [28]). If $p \in \mathfrak{R}$, then the following conclusions hold:

(i) $e_{p}(t, t) \equiv 1$, and $e_{0}(t, s) \equiv 1$, 
(ii) $e_{p}(\sigma(t), s)=(1+\mu(t) p(t)) e_{p}(t, s)$,

(iii) $e_{p}(t, s)=1 / e_{p}(s, t)=e_{\ominus p}(s, t)$,

where $\ominus p=-p /(1+\mu p)$.

Theorem 2.15 (see [28]). If $p \in \mathfrak{R}$, and fix $t_{0} \in \mathbb{T}$, then the exponential function $e_{p}\left(t, t_{0}\right)$ is the unique solution of the following initial value problem:

$$
\begin{gathered}
y^{\Delta}(t)=p(t) y(t) \\
y\left(t_{0}\right)=1
\end{gathered}
$$

For more details about the calculus of time scales, we advise to refer to [29].

\section{Main Results}

In the rest of this paper, for the sake of convenience, we denote $\mathbb{T}_{0}=\left[t_{0}, \infty\right) \cap \mathbb{T}$, where $t_{0} \in \mathbb{T}$, and always assume $\mathbb{T}_{0} \subset \mathbb{T}^{\mathcal{K}}$.

Lemma 3.1 (see [30, Theorem 2.2]). Let $t_{0} \in \mathbb{T}^{\mathcal{K}}$ and $\omega: \mathbb{T} \times \mathbb{T}^{\mathcal{K}} \rightarrow \mathbb{R}$ be continuous at $(t, t)$, where $t \geq t_{0}, t \in \mathbb{T}^{\mathcal{K}}$ with $t>t_{0}$. Assume that $\omega^{\Delta}(t, \cdot)$ is $r d$-continuous on $\left[t_{0}, \sigma(t)\right]$. If for any $\varepsilon>0$, there exists a neighborhood $U$ of $t$, independent of $\tau \in\left[t_{0}, \sigma(t)\right]$, such that

$$
\left|\omega(\sigma(t), \tau)-\omega(s, \tau)-\omega^{\Delta}(t, \tau)(\sigma(t)-s)\right| \leq \varepsilon|\sigma(t)-s| \quad \forall s \in U
$$

where $\omega^{\Delta}$ denotes the derivative of $\omega$ with respect to the first variable, then

$$
g(t):=\int_{t_{0}}^{t} \omega(t, \tau) \Delta \tau
$$

implies

$$
g^{\Delta}(t)=\int_{t_{0}}^{t} \omega^{\Delta}(t, \tau) \Delta \tau+\omega(\sigma(t), t)
$$

Theorem 3.2. Suppose $u \in C_{\mathrm{rd}}\left(\mathbb{T}_{0}, \mathbb{R}_{+}\right), \omega \in\left(\mathbb{R}_{+}, \mathbb{R}_{+}\right)$with $\omega(u)>0$ for $u>0$, and $\omega$ is nondecreasing. $\tau_{i} \in\left(\mathbb{T}_{0}, \mathbb{T}\right)$ with $\tau_{i}(t) \leq t, i=1,2$, and $-\infty<\alpha=\inf \left\{\min \left\{\tau_{i}(t), i=1,2\right\}, t \in\right.$ $\left.\mathbb{T}_{0}\right\} \leq t_{0} . f, g \in C_{\mathrm{rd}}\left([\alpha, \infty) \cap \mathbb{T}, \mathbb{R}_{+}\right) . p, q, C$ are constants, and $p>q>0, C \geq 0$. If for $t \in \mathbb{T}_{0}, u(t)$ satisfies the following inequality:

$$
u^{p}(t) \leq C^{p /(p-q)}+\frac{p}{p-q} \int_{t_{0}}^{t}\left[f\left(\tau_{1}(s)\right) u^{q}\left(\tau_{1}(s)\right) \omega\left(u\left(\tau_{1}(s)\right)\right)+g\left(\tau_{2}(s)\right) u^{q}\left(\tau_{2}(s)\right)\right] \Delta s
$$


with the initial condition

$$
\begin{gathered}
u(t)=\phi(t), \quad t \in\left[\alpha, t_{0}\right] \cap \mathbb{T}, \\
\phi\left(\tau_{i}(t)\right) \leq C^{1 /(p-q)}, \quad \forall t \in \mathbb{T}_{0}, \tau_{i}(t) \leq t_{0}, i=1,2,
\end{gathered}
$$

where $\phi \in C_{\mathrm{rd}}\left(\left[\alpha, t_{0}\right] \cap \mathbb{T}, \mathbb{R}_{+}\right)$, then,

$$
u(t) \leq\left\{G^{-1}\left[G\left(C+\int_{t_{0}}^{t} g\left(\tau_{2}(s)\right) \Delta s\right)+\int_{t_{0}}^{t} f\left(\tau_{1}(s)\right) \Delta s\right]\right\}^{1 /(p-q)}, \quad t \in \mathbb{T}_{0}
$$

where $G(x)=\int_{1}^{x} 1 / \omega\left(r^{1 /(p-q)}\right) d r, x>0$ with $G(\infty)=\infty$, and $G^{-1}$ is the inverse of $G$.

Proof. Assume $C>0$. Denote the right side of (3.4) by $z(t)$. Then

$$
u(t) \leq z^{1 / p}(t), \quad t \in \mathbb{T}_{0}
$$

If $\tau_{i}(t) \geq t_{0}$, for $t \in \mathbb{T}_{0}$, since $\tau_{i}(t) \leq t$, then $\tau_{i}(t) \in \mathbb{T}_{0}$, and from (3.7) we have

$$
u\left(\tau_{i}(t)\right) \leq z^{1 / p}\left(\tau_{i}(t)\right) \leq z^{1 / p}(t), \quad i=1,2
$$

If $\tau_{i}(t) \leq t_{0}$, from (3.5) we obtain

$$
u\left(\tau_{i}(t)\right)=\phi\left(\tau_{i}(t)\right) \leq C^{1 /(p-q)} \leq z^{1 / p}(t), \quad i=1,2 .
$$

So from (3.8) and (3.9) we always have

$$
u\left(\tau_{i}(t)\right) \leq z^{1 / p}(t), \quad i=1,2, t \in \mathbb{T}_{0} .
$$

Furthermore,

$$
\begin{aligned}
z^{\Delta}(t) & =\frac{p}{p-q}\left[f\left(\tau_{1}(t)\right) u^{q}\left(\tau_{1}(t)\right) \omega\left(u\left(\tau_{1}(t)\right)\right)+g\left(\tau_{2}(t)\right) u^{q}\left(\tau_{2}(t)\right)\right] \\
& \leq \frac{p}{p-q}\left[f\left(\tau_{1}(t)\right) z^{q / p}(t) \omega\left(z^{1 / p}(t)\right)+g\left(\tau_{2}(t)\right) z^{q / p}(t)\right]
\end{aligned}
$$

that is,

$$
\frac{z^{\Delta}(t)}{z^{q / p}(t)} \leq \frac{p}{p-q}\left[f\left(\tau_{1}(t)\right) \omega\left(z^{1 / p}(t)\right)+g\left(\tau_{2}(t)\right)\right]
$$


According to [29, Theorem 1.90], considering $z^{\Delta}(t) \geq 0$, we have

$$
\begin{aligned}
\left(\frac{p}{p-q} z^{(p-q) / p}(t)\right)^{\Delta} & =z^{\Delta}(t) \int_{0}^{1}\left[z(t)+h \mu(t) z^{\Delta}(t)\right]^{-q / p} d h \\
& =\frac{z^{\Delta}(t)}{z^{q / p}(t)} \int_{0}^{1}\left[1+h \mu(t) \frac{z^{\Delta}(t)}{z(t)}\right]^{-q / p} d h \\
& \leq \frac{z^{\Delta}(t)}{z^{q / p}(t)}
\end{aligned}
$$

Combining (3.12) and (3.13), we obtain

$$
\left(z^{(p-q) / p}(t)\right)^{\Delta} \leq f\left(\tau_{1}(t)\right) \omega\left(z^{1 / p}(t)\right)+g\left(\tau_{2}(t)\right)
$$

Setting $t=s$ in (3.14), an integration with respect to $s$ from $t_{0}$ to $t$ yields

$$
z^{(p-q) / p}(t)-z^{(p-q) / p}\left(t_{0}\right) \leq \int_{t_{0}}^{t}\left[f\left(\tau_{1}(s)\right) \omega\left(z^{1 / p}(s)\right)+g\left(\tau_{2}(s)\right)\right] \Delta s .
$$

Since $z\left(t_{0}\right)=C^{p /(p-q)}$, then (3.15) implies

$$
z(t) \leq\left\{C+\int_{t_{0}}^{t}\left[f\left(\tau_{1}(s)\right) \omega\left(z^{1 / p}(s)\right)+g\left(\tau_{2}(s)\right)\right] \Delta s\right\}^{p /(p-q)} .
$$

Fix $T \in \mathbb{T}_{0}$, and let $t \in\left[t_{0}, T\right] \cap \mathbb{T}$. Then,

$$
z(t) \leq\left\{C+\int_{t_{0}}^{T} g\left(\tau_{2}(s)\right) \Delta s+\int_{t_{0}}^{t} f\left(\tau_{1}(s)\right) \omega\left(z^{1 / p}(s)\right) \Delta s\right\}^{p /(p-q)} .
$$

Denote $v(t)$ by $C+\int_{t_{0}}^{T} g\left(\tau_{2}(s)\right) \Delta s+\int_{t_{0}}^{t} f\left(\tau_{1}(s)\right) \omega\left(z^{1 / p}(s)\right) \Delta s$. Then,

$$
\begin{gathered}
z(t) \leq v^{p /(p-q)}(t), \quad t \in\left[t_{0}, T\right] \cap \mathbb{T}, \\
v^{\Delta}(t)=f\left(\tau_{1}(t)\right) \omega\left(z^{1 / p}(t)\right) \leq f\left(\tau_{1}(t)\right) \omega\left(v^{1 /(p-q)}(t)\right),
\end{gathered}
$$

that is,

$$
\frac{v^{\Delta}(t)}{\omega\left(v^{1 /(p-q)}(t)\right)} \leq f\left(\tau_{1}(t)\right)
$$


On the other hand, for $t \in\left[t_{0}, T\right] \cap \mathbb{T}$, if $\sigma(t)>t$, then

$$
\begin{aligned}
{[G(v(t))]^{\Delta} } & =\frac{G(v(\sigma(t)))-G(v(t))}{\sigma(t)-t}=\frac{1}{\sigma(t)-t} \int_{v(t)}^{v(\sigma(t))} \frac{1}{\omega\left(r^{1 /(p-q)}\right)} d r \\
& \leq \frac{v(\sigma(t))-v(t)}{\sigma(t)-t} \frac{1}{\omega\left(v^{1 /(p-q)}(t)\right)}=\frac{v^{\Delta}(t)}{\omega\left(v^{1 /(p-q)}(t)\right)} .
\end{aligned}
$$

If $\sigma(t)=t$, then

$$
\begin{aligned}
{[G(v(t))]^{\Delta} } & =\lim _{s \rightarrow t} \frac{G(v(t))-G(v(s))}{t-s}=\lim _{s \rightarrow t} \frac{1}{t-s} \int_{v(s)}^{v(t)} \frac{1}{\omega\left(r^{1 /(p-q)}\right)} d r \\
& =\lim _{s \rightarrow t} \frac{v(t)-v(s)}{t-s} \frac{1}{\omega\left(\xi^{1 /(p-q)}\right)}=\frac{v^{\Delta}(t)}{\omega\left(v^{1 /(p-q)}(t)\right)}
\end{aligned}
$$

where $\xi$ lies between $v(s)$ and $v(t)$. So from (3.21) and (3.22) we always have

$$
[G(v(t))]^{\Delta} \leq \frac{v^{\Delta}(t)}{\omega\left(v^{1 /(p-q)}(t)\right)}
$$

Combining (3.20) and (3.23), we deduce

$$
[G(v(t))]^{\Delta} \leq f\left(\tau_{1}(t)\right)
$$

Setting $t=s$ in (3.24), an integration with respect to $s$ from $t_{0}$ to $t$ yields

$$
G(v(t))-G\left(v\left(t_{0}\right)\right) \leq \int_{t_{0}}^{t} f\left(\tau_{1}(s)\right) \Delta s
$$

Considering $v\left(t_{0}\right)=C+\int_{t_{0}}^{T} g\left(\tau_{2}(s)\right) \Delta s$, and $G$ is increasing, then we obtain

$$
v(t) \leq G^{-1}\left[G\left(C+\int_{t_{0}}^{T} g\left(\tau_{2}(s)\right) \Delta s\right)+\int_{t_{0}}^{t} f\left(\tau_{1}(s)\right) \Delta s\right], \quad t \in\left[t_{0}, T\right] \cap \mathbb{T} .
$$

Combining (3.7), (3.18), and (3.26), we have

$$
u(t) \leq\left\{G^{-1}\left[G\left(C+\int_{t_{0}}^{T} g\left(\tau_{2}(s)\right) \Delta s\right)+\int_{t_{0}}^{t} f\left(\tau_{1}(s)\right) \Delta s\right]\right\}^{1 /(p-q)}, \quad t \in\left[t_{0}, T\right] \cap \mathbb{T} .
$$

Setting $t=T$ in (3.27), considering that $T \in \mathbb{T}_{0}$ is selected arbitrarily, after substituting $T$ with $t$, we get the desired result.

If $C=0$, then we carry out the process above with $C$ replaced by $\varepsilon$, where $\varepsilon>0$, and after letting $\varepsilon \rightarrow 0$, we also get the desired result. So the proof is complete. 
Remark 3.3. If we take $\mathbb{T}=\mathbb{R}, t_{0}=0$, then Theorem 3.2 reduces to [31, Theorem 2.1]. If $\mathbb{T}=\mathbb{R}$, $t_{0}=0, p=2, q=1$, then Theorem 3.2 reduces to [32, Theorem 1]. If $\mathbb{T}=\mathbb{R}, t_{0}=0, p=2, q=1$, $\tau_{1}(t)=\tau_{2}(t)=t$, then Theorem 3.2 reduces to [33, Theorem 3(a6)]. If we take $\mathbb{T}=\mathbb{Z}, t_{0}=0$, $p=2, q=1, \tau_{1}(t)=\tau_{2}(t)=t$, then Theorem 3.2 reduces to [33, Theorem 6(b6)].

Theorem 3.4. Suppose $u, a \in C_{\mathrm{rd}}\left(\mathbb{T}_{0}, \mathbb{R}_{+}\right)$, and $a$ is nondecreasing. $\alpha, \phi, \tau_{i}, i=1,2$ are defined as in Theorem 3.2. $f, h, f_{t}^{\Delta}, h_{t}^{\Delta} \in C_{\mathrm{rd}}\left(\mathbb{T}_{0} \times([\alpha, \infty) \cap \mathbb{T}), \mathbb{R}_{+}\right), g, d, g_{t}^{\Delta}, d_{t}^{\Delta} \in C_{\mathrm{rd}}\left(\mathbb{T}_{0}^{2}, \mathbb{R}_{+}\right)$, where $f_{t}^{\Delta}, h_{t}^{\Delta}, g_{t}^{\Delta}, d_{t}^{\Delta}$ denote the delta derivative of $f, h, g, d$ with respect to the first variable. If for $t \in \mathbb{T}_{0}$, $u(t)$ satisfies the following inequality:

$$
\begin{aligned}
u(t) \leq & a(t)+\int_{t_{0}}^{t}\left[f\left(t, \tau_{1}(s)\right) u\left(\tau_{1}(s)\right)+g(t, s) u(s)\right] \Delta s \\
& +\int_{t_{0}}^{t} h\left(t, \tau_{2}(s)\right) u\left(\tau_{2}(s)\right) \Delta s \int_{t_{0}}^{t} d(t, s) u(s) \Delta s
\end{aligned}
$$

with the initial condition

$$
\begin{gathered}
u(t)=\phi(t), \quad t \in\left[\alpha, t_{0}\right] \cap \mathbb{T}, \\
\phi\left(\tau_{i}(t)\right) \leq a(t), \quad \forall t \in \mathbb{T}_{0}, \tau_{i}(t) \leq t_{0}, \quad i=1,2,
\end{gathered}
$$

then

$$
u(t) \leq \frac{a(t) e_{\ominus\left(-F_{1}\right)}\left(t, t_{0}\right)}{1+a(t) \int_{t_{0}}^{t} e_{\ominus\left(-F_{1}\right)}\left(\sigma(s), t_{0}\right) F_{2}(s) \Delta s}, \quad t \in \mathbb{T}_{0}
$$

provided that $1+a(t) \int_{t_{0}}^{t} e_{\ominus\left(-F_{1}\right)}\left(\sigma(s), t_{0}\right) F_{2}(s) \Delta s>0$ and $1-\mu(t) F_{1}(t)>0$ for $\forall t \in \mathbb{T}_{0}$, where

$$
F_{1}(t)=\left\{\int_{t_{0}}^{t}\left[f\left(t, \tau_{1}(s)\right)+g(t, s)\right] \Delta s\right\}^{\Delta}, \quad F_{2}(t)=\left\{\int_{t_{0}}^{t} h\left(t, \tau_{2}(s)\right) \Delta s \int_{t_{0}}^{t} d(t, s) \Delta s\right\}^{\Delta} .
$$

Proof. Assume $a(0)>0$. Fix $T \in \mathbb{T}_{0}$, and let $t \in\left[t_{0}, T\right] \cap \mathbb{T}$. If the right side of $(3.28)$ is $a(t)+z(t)$, then

$$
u(t) \leq a(t)+z(t)
$$

and similar to the process of (3.8)-(3.10), we have

$$
u\left(\tau_{i}(t)\right) \leq a(t)+z(t), \quad i=1,2
$$


Furthermore, by Lemma 3.1 and Theorem 2.9(ii)

$$
\begin{aligned}
z^{\Delta}(t)= & \int_{t_{0}}^{t}\left[f_{t}^{\Delta}\left(t, \tau_{1}(s)\right) u\left(\tau_{1}(s)\right)+g_{t}^{\Delta}(t, s) u(s)\right] \Delta s+f\left(\sigma(t), \tau_{1}(t)\right) u\left(\tau_{1}(t)\right)+g(\sigma(t), t) u(t) \\
& +\left[\int_{t_{0}}^{t} h_{t}^{\Delta}\left(t, \tau_{2}(s)\right) u\left(\tau_{2}(s)\right) \Delta s+h\left(\sigma(t), \tau_{2}(t)\right) u\left(\tau_{2}(t)\right)\right] \int_{t_{0}}^{\sigma(t)} d(\sigma(t), s) u(s) \Delta s \\
& +\int_{t_{0}}^{t} h\left(t, \tau_{2}(s)\right) u\left(\tau_{2}(s)\right) \Delta s\left[\int_{t_{0}}^{t} d_{t}^{\Delta}(t, s) u(s) \Delta s+d(\sigma(t), t)\right] \\
\leq & {[a(t)+z(t)]\left\{\int_{t_{0}}^{t}\left[f_{t}^{\Delta}\left(t, \tau_{1}(s)\right)+g_{t}^{\Delta}(t, s)\right] \Delta s+f\left(\sigma(t), \tau_{1}(t)\right)+g(\sigma(t), t)\right\} } \\
& +[a(t)+z(t)]^{2}\left\{\left[\int_{t_{0}}^{t} h_{t}^{\Delta}\left(t, \tau_{2}(s)\right) \Delta s+h\left(\sigma(t), \tau_{2}(t)\right)\right] \int_{t_{0}}^{\sigma(t)} d(\sigma(t), s) \Delta s\right. \\
\leq & {[a(T)+z(t)]\left\{\int_{t_{0}}^{t}\left[f\left(t, \tau_{1}(s)\right)+g(t, s)\right] \Delta s \int_{t_{0}}^{t} h\left(t, \tau_{2}(s)\right) \Delta s\left[\int_{t_{0}}^{t} d_{t}^{\Delta}(t, s) \Delta s+d(\sigma(t), t)\right]\right\} } \\
& +[a(T)+z(t)]^{2}\left\{\int_{t_{0}}^{t} h\left(t, \tau_{2}(s)\right) \Delta s \int_{t_{0}}^{t} d(t, s) \Delta s\right\},
\end{aligned}
$$

that is,

$$
\begin{aligned}
\frac{z^{\Delta}(t)}{[a(T)+z(t)]^{2}} \leq & \frac{1}{a(T)+z(t)}\left\{\int_{t_{0}}^{t}\left[f\left(t, \tau_{1}(s)\right)+g(t, s)\right] \Delta s\right\}^{\Delta} \\
& +\left\{\int_{t_{0}}^{t} h\left(t, \tau_{2}(s)\right) \Delta s \int_{t_{0}}^{t} d(t, s) \Delta s\right\}^{\Delta} \\
\leq & \frac{1}{a(T)+z(t)} F_{1}(t)+F_{2}(t)
\end{aligned}
$$

where $F_{1}, F_{2}$ are defined in (3.31).

Considering $z^{\Delta}(t) \geq 0$, from (3.35), we deduce

$$
\frac{z^{\Delta}(t)}{[a(T)+z(t)][a(T)+z(\sigma(t))]} \leq \frac{z^{\Delta}(t)}{[a(T)+z(t)]^{2}} \leq \frac{1}{a(T)+z(t)} F_{1}(t)+F_{2}(t) .
$$


Let $v(t)=1 /(a(T)+z(t))$. Then, by Theorem 2.9(ii), $v^{\Delta}(t)=-z^{\Delta}(t) /[a(T)+z(t)][a(T)+$ $z(\sigma(t))]$, and (3.36) implies

$$
v^{\Delta}(t)+v(t) F_{1}(t) \geq F_{2}(t)
$$

On the other hand, since $1-\mu(t) F_{1}(t)>0$, then $1+\mu(t)\left[\ominus\left(-F_{1}\right)(t)\right]=1 /\left(1-\mu(t) F_{1}(t)\right)>0$. So $\ominus\left(-F_{1}\right) \in \mathfrak{R}_{+}$, and $e_{\ominus\left(-F_{1}\right)}\left(t, t_{0}\right)>0, \forall t \in \mathbb{T}_{0}$. By Theorem 2.14(i), we have $e_{\ominus\left(-F_{1}\right)}\left(t_{0}, t_{0}\right)=1$. Furthermore, by a combination of Theorem 2.9(ii), Theorems 2.15, and 2.14, we obtain

$$
\begin{aligned}
{\left[v(t) e_{\ominus\left(-F_{1}\right)}\left(t, t_{0}\right)\right]^{\Delta} } & =\left[e_{\ominus\left(-F_{1}\right)}\left(t, t_{0}\right)\right]^{\Delta} v(t)+e_{\ominus\left(-F_{1}\right)}\left(\sigma(t), t_{0}\right) v^{\Delta}(t) \\
& =\left(\ominus\left(-F_{1}\right)\right)(t) e_{\ominus\left(-F_{1}\right)}\left(t, t_{0}\right) v(t)+e_{\ominus\left(-F_{1}\right)}\left(\sigma(t), t_{0}\right) v^{\Delta}(t) \\
& =e_{\ominus\left(-F_{1}\right)}\left(\sigma(t), t_{0}\right)\left[\frac{\left(\ominus\left(-F_{1}\right)\right)(t)}{1+\mu(t)\left(\ominus\left(-F_{1}\right)\right)(t)} v(t)+v^{\Delta}(t)\right] \\
& =e_{\ominus\left(-F_{1}\right)}\left(\sigma(t), t_{0}\right)\left[v^{\Delta}(t)+F_{1}(t) v(t)\right] .
\end{aligned}
$$

Combining (3.37) and (3.38), we deduce

$$
\left[v(t) e_{\ominus\left(-F_{1}\right)}\left(t, t_{0}\right)\right]^{\Delta} \geq e_{\ominus\left(-F_{1}\right)}\left(\sigma(t), t_{0}\right) F_{2}(t)
$$

Setting $t=s$ in (3.39), an integration with respect to $s$ from $t_{0}$ to $t$ yields

$$
v(t) e_{\ominus\left(-F_{1}\right)}\left(t, t_{0}\right)-v\left(t_{0}\right) e_{\ominus\left(-F_{1}\right)}\left(t_{0}, t_{0}\right) \geq \int_{t_{0}}^{t} e_{\ominus\left(-F_{1}\right)}\left(\sigma(s), t_{0}\right) F_{2}(s) \Delta s
$$

Considering $v\left(t_{0}\right)=1 / a(T)$, it is then followed by

$$
v(t) \geq \frac{1+a(T) \int_{t_{0}}^{t} e_{\ominus\left(-F_{1}\right)}\left(\sigma(s), t_{0}\right) F_{2}(s) \Delta s}{a(T) e_{\ominus\left(-F_{1}\right)}\left(t, t_{0}\right)},
$$

and furthermore

$$
a(T)+z(t) \leq \frac{a(T) e_{\ominus\left(-F_{1}\right)}\left(t, t_{0}\right)}{1+a(T) \int_{t_{0}}^{t} e_{\ominus\left(-F_{1}\right)}\left(\sigma(s), t_{0}\right) F_{2}(s) \Delta s} .
$$

Combining (3.32) and (3.42), we obtain

$$
u(t) \leq \frac{a(T) e_{\ominus\left(-F_{1}\right)}\left(t, t_{0}\right)}{1+a(T) \int_{t_{0}}^{t} e_{\ominus\left(-F_{1}\right)}\left(\sigma(s), t_{0}\right) F_{2}(s) \Delta s}, \quad t \in\left[t_{0}, T\right] \cap \mathbb{T} .
$$

Setting $t=T$ in (3.43), since $T \in \mathbb{T}_{0}$ is selected arbitrarily, after substituting $T$ with $t$, we get the desired result. 
If $a(0)=0$, then we carry out the process above with $a(t)$ replaced by $a(t)+\varepsilon$, and after letting $\varepsilon \rightarrow 0$, we also get the desired result. So the proof is complete.

Remark 3.5. If we take $\mathbb{T}=\mathbb{R}, t_{0}=0$, then Theorem 3.4 reduces to [34, Corollary 2.5]. If $\mathbb{T}=\mathbb{R}$, $t_{0}=0, \tau_{1}(t)=\tau_{2}(t)=t, a(t) \equiv C$, where $C$ is a nonnegative constant, and $f(t, s), g(t, s), h(t, s)$ are replaced by $f(s), g(s), h(s)$, then Theorem 3.4 reduces to [35, Theorem 1]. If we take $\mathbb{T}=\mathbb{Z}, t_{0}=0, \tau_{1}(t)=\tau_{2}(t)=t,(t) \equiv C$, where $C$ is a nonnegative constant, and $f(t, s),(t, s)$, $h(t, s)$ are replaced by $f(s), g(s), h(s)$, then Theorem 3.4 reduces to [35, Theorem 5].

Next we will study the delay integral inequality on time scales with the following form

$$
\begin{aligned}
u^{p}(t) \leq & C^{p /(p-q)}+\frac{p}{p-q} \int_{t_{0}}^{t} f\left(\tau_{1}(s)\right) u^{p}\left(\tau_{1}(s)\right) \Delta s \\
& +\frac{p}{p-q} \int_{t_{0}}^{t}\left[f\left(\tau_{1}(s)\right) u^{q}\left(\tau_{1}(s)\right) \int_{t_{0}}^{s} g\left(\tau_{1}(\xi)\right) u^{p-q}\left(\tau_{1}(\xi)\right) \Delta \xi+h\left(\tau_{2}(s)\right) u^{q}\left(\tau_{2}(s)\right)\right] \Delta s,
\end{aligned}
$$

where $u, f, g, p, q, C, \alpha, \phi, \tau_{i}, i=1,2$ are defined as in Theorem 3.2, and $h \in C_{\mathrm{rd}}\left([\alpha, \infty) \cap \mathbb{T}, \mathbb{R}_{+}\right)$.

Lemma 3.6. Suppose $u, f, g, \tau_{1}$ are defined as in Theorem 3.2, and $(f+g)\left(\tau_{1}(\cdot)\right) \in \mathfrak{R}_{+}$, then for $t \in \mathbb{T}_{0}$,

$$
u(t) \leq 1+\int_{t_{0}}^{t} f\left(\tau_{1}(s)\right) u(s) \Delta s+\int_{t_{0}}^{t}\left[f\left(\tau_{1}(s)\right) \int_{t_{0}}^{s} g\left(\tau_{1}(\xi)\right) u(\xi) \Delta \xi\right] \Delta s
$$

implies

$$
u(t) \leq 1+\int_{t_{0}}^{t} f\left(\tau_{1}(s)\right) e_{(f+g)\left(\tau_{1}(\cdot)\right)}\left(s, t_{0}\right) \Delta s
$$

Proof. Denote the right side of (3.46) by $z(t)$. Then, $u(t) \leq z(t), t \in \mathbb{T}_{0}$, and

$$
\begin{aligned}
z^{\Delta}(t) & =f\left(\tau_{1}(t)\right) u(t)+f\left(\tau_{1}(t)\right) \int_{t_{0}}^{t} g\left(\tau_{1}(\xi)\right) u(\xi) \Delta \xi \\
& \leq f\left(\tau_{1}(t)\right) z(t)+f\left(\tau_{1}(t)\right) \int_{t_{0}}^{t} g\left(\tau_{1}(\xi)\right) z(\xi) \Delta \xi .
\end{aligned}
$$

Let $m(t)=z(t)+\int_{t_{0}}^{t} g\left(\tau_{1}(\xi)\right) z(\xi) \Delta \xi$. Then, $z(t) \leq m(t)$, and $z^{\Delta}(t) \leq f\left(\tau_{1}(t)\right) m(t)$. Furthermore,

$$
m^{\Delta}(t)=z^{\Delta}(t)+g\left(\tau_{1}(t)\right) z(t) \leq f\left(\tau_{1}(t)\right) m(t)+g\left(\tau_{1}(t)\right) m(t) .
$$

Since $(f+g)\left(\tau_{1}(\cdot)\right) \in \mathfrak{R}_{+}$, by $\left[28\right.$, Theorem 5.4], we have $m(t) \leq e_{(f+g)\left(\tau_{1}(\cdot)\right)}\left(t, t_{0}\right)$. So,

$$
z^{\Delta}(t) \leq f\left(\tau_{1}(t)\right) e_{(f+g)\left(\tau_{1}(\cdot)\right)}\left(t, t_{0}\right)
$$


Using $z\left(t_{0}\right)=1$, an integration for (3.49) from $t_{0}$ to $t$ yields

$$
z(t) \leq 1+\int_{t_{0}}^{t} f\left(\tau_{1}(s)\right) e_{(f+g)\left(\tau_{1}(\cdot)\right)}\left(s, t_{0}\right) \Delta s
$$

which confirms the desired inequality. So the proof is complete.

Theorem 3.7. If for $t \in \mathbb{T}_{0}, u(t)$ satisfies the inequality (3.44) with the initial condition (3.5), then

$$
u(t) \leq\left\{J(t)\left[1+\int_{t_{0}}^{t} f\left(\tau_{1}(s)\right) e_{(f+g)\left(\tau_{1}(\cdot)\right)}\left(s, t_{0}\right) \Delta s\right]\right\}^{1 /(p-q)}, \quad t \in \mathbb{T}_{0}
$$

provided $(f+g)\left(\tau_{1}(\cdot)\right) \in \mathfrak{R}_{+}$, where

$$
J(t)=C+\int_{t_{0}}^{t} h\left(\tau_{2}(s)\right) \Delta s
$$

Proof. Let

$$
\begin{aligned}
& z(t)=(C+\varepsilon)^{p /(p-q)} \\
& +\frac{p}{p-q} \int_{t_{0}}^{t}\left[f\left(\tau_{1}(s)\right) u^{p}\left(\tau_{1}(s)\right) \Delta s+\frac{p}{p-q} \int_{t_{0}}^{t} f\left(\tau_{1}(s)\right) u^{q}\left(\tau_{1}(s)\right)\right. \\
& \left.\quad \quad \quad \int_{t_{0}}^{s} g\left(\tau_{1}(\xi)\right) u^{p-q}\left(\tau_{1}(\xi)\right) \Delta \xi+h\left(\tau_{2}(s)\right) u^{q}\left(\tau_{2}(s)\right)\right] \Delta s,
\end{aligned}
$$

where $\varepsilon>0$ is an arbitrary small constant. Then,

$$
u(t) \leq z^{1 / p}(t), \quad t \in \mathbb{T}_{0}
$$

and similar to (3.8)-(3.10)

$$
u\left(\tau_{i}(t)\right) \leq z^{1 / p}(t), \quad i=1,2, t \in \mathbb{T}_{0} .
$$


Furthermore,

$$
\begin{aligned}
z^{\Delta}(t)= & \frac{p}{p-q} f\left(\tau_{1}(t)\right) u^{p}\left(\tau_{1}(t)\right) \\
& +\frac{p}{p-q}\left[f\left(\tau_{1}(t)\right) u^{q}\left(\tau_{1}(t)\right) \int_{t_{0}}^{t} g\left(\tau_{1}(\xi)\right) u^{p-q}\left(\tau_{1}(\xi)\right) \Delta \xi+h\left(\tau_{2}(t)\right) u^{q}\left(\tau_{2}(t)\right)\right] \\
\leq & \frac{p}{p-q} f\left(\tau_{1}(t)\right) z(t) \\
& +\frac{p}{p-q}\left[f\left(\tau_{1}(t)\right) z^{q / p}(t) \int_{t_{0}}^{t} g\left(\tau_{1}(\xi)\right) z^{(p-q) / p}(\xi) \Delta \xi+h\left(\tau_{2}(t)\right) z^{q / p}(t)\right] .
\end{aligned}
$$

Using (3.13), we obtain $\left((p /(p-q)) z^{(p-q) / p}(t)\right)^{\Delta} \leq\left(z^{\Delta}(t) / z^{q / p}(t)\right)$. So (3.56) implies

$$
\left(z^{(p-q) / p}(t)\right)^{\Delta} \leq f\left(\tau_{1}(t)\right) z^{(p-q) / p}(t)+f\left(\tau_{1}(t)\right) \int_{t_{0}}^{t} g\left(\tau_{1}(\xi)\right) z^{(p-q) / p}(\xi) \Delta \xi+h\left(\tau_{2}(t)\right)
$$

Considering $z\left(t_{0}\right)=(C+\varepsilon)^{p /(p-q)}$, an integration for (3.57) from $t_{0}$ to $t$ yields

$$
\begin{aligned}
z^{(p-q) / p}(t) \leq & C+\varepsilon+\int_{t_{0}}^{t} f\left(\tau_{1}(s)\right) z^{(p-q) / p}(s) \Delta s \\
& +\int_{t_{0}}^{t}\left[f\left(\tau_{1}(s)\right) \int_{t_{0}}^{s} g\left(\tau_{1}(\xi)\right) z^{(p-q) / p}(\xi) \Delta \xi+h\left(\tau_{2}(s)\right)\right] \Delta s \\
= & J_{\varepsilon}(t)+\int_{t_{0}}^{t} f\left(\tau_{1}(s)\right) z^{(p-q) / p}(s) \Delta s \\
& +\int_{t_{0}}^{t}\left[f\left(\tau_{1}(s)\right) \int_{t_{0}}^{s} g\left(\tau_{1}(\xi)\right) z^{(p-q) / p}(\xi) \Delta \xi\right] \Delta s,
\end{aligned}
$$

where $J_{\varepsilon}(t)=J(t)+\varepsilon$, and $J(t)$ is defined in (3.52). Then,

$$
\frac{z^{(p-q) / p}(t)}{J_{\varepsilon}(t)} \leq 1+\int_{t_{0}}^{t} f\left(\tau_{1}(s)\right) \frac{z^{(p-q) / p}(s)}{J_{\varepsilon}(s)} \Delta s+\int_{t_{0}}^{t}\left[f\left(\tau_{1}(s)\right) \int_{t_{0}}^{s} g\left(\tau_{1}(\xi)\right) \frac{z^{(p-q) / p}(\xi)}{J_{\varepsilon}(\xi)} \Delta \xi\right] \Delta s .
$$

Denote $z^{(p-q) / p}(t) / J_{\varepsilon}(t)=v(t)$. Then,

$$
v(t) \leq 1+\int_{t_{0}}^{t} f\left(\tau_{1}(s)\right) v(s) \Delta s+\int_{t_{0}}^{t}\left[f\left(\tau_{1}(s)\right) \int_{t_{0}}^{s} g\left(\tau_{1}(\xi)\right) v(\xi) \Delta \xi\right] \Delta s .
$$


A suitable application of Lemma 3.6 to (3.60) yields

$$
v(t) \leq 1+\int_{t_{0}}^{t} f\left(\tau_{1}(s)\right) e_{(f+g)\left(\tau_{1}(\cdot)\right)}\left(s, t_{0}\right) \Delta s .
$$

Combining (3.54) and (3.61), we obtain

$$
u(t) \leq\left\{J_{\varepsilon}(t)\left[1+\int_{t_{0}}^{t} f\left(\tau_{1}(s)\right) e_{(f+g)\left(\tau_{1}(\cdot)\right)}\left(s, t_{0}\right) \Delta s\right]\right\}^{1 /(p-q)} .
$$

After letting $\varepsilon \rightarrow 0$, we get the desired result. So the proof is complete.

Remark 3.8. If we take $\mathbb{T}=\mathbb{R}, t_{0}=0, p=2, q=1, \tau_{1}(t)=\tau_{2}(t)=t$, then Theorem 3.7 reduces to [33, Theorem 1(a2)]. If we take $\mathbb{T}=\mathbb{Z}, t_{0}=0, p=2, q=1, \tau_{1}(t)=\tau_{2}(t)=t$, then Theorem 3.7 reduces to [33, Theorem $4(\mathrm{~b} 2)]$.

Now we present a more general inequality than in Theorem 3.7.

Theorem 3.9. Suppose $u, f, g, \omega, p, q, C, \alpha, \phi, \tau_{i}, i=1,2$ are defined as in Theorem 3.2, and $h \in$ $C_{\mathrm{rd}}\left([\alpha, \infty) \cap \mathbb{T}, \mathbb{R}_{+}\right)$. If for $t \in \mathbb{T}_{0}, u(t)$ satisfies the following inequality:

$$
\begin{gathered}
u^{p}(t) \leq C^{p /(p-q)}+\frac{p}{p-q} \int_{t_{0}}^{t} f\left(\tau_{1}(s)\right) u^{p}\left(\tau_{1}(s)\right) \Delta s+\frac{p}{p-q} \int_{t_{0}}^{t}\left[f\left(\tau_{1}(s)\right) u^{q}\left(\tau_{1}(s)\right)\right. \\
\left.\times \int_{t_{0}}^{s} g\left(\tau_{1}(\xi)\right) \omega\left(u\left(\tau_{1}(\xi)\right)\right) \Delta \xi+h\left(\tau_{2}(s)\right) u^{q}\left(\tau_{2}(s)\right)\right] \Delta s
\end{gathered}
$$

with the initial condition (3.5), then for $t \in \mathbb{T}_{0}$,

$$
u(t) \leq\left\{J(t)+\int_{t_{0}}^{t} f\left(\tau_{1}(s)\right) \tilde{G}^{-1}\left\{\tilde{G}(J(s))+\int_{t_{0}}^{s}\left[f\left(\tau_{1}(\xi)\right)+g\left(\tau_{1}(\xi)\right)\right] \Delta \xi\right\} \Delta s\right\}^{1 /(p-q)},
$$

where $J(t)$ is defined as in Theorem 3.7, and

$$
\tilde{G}(x)=\int_{1}^{x} \frac{1}{r+\omega\left(r^{1 /(p-q)}\right)} d r, \quad x>0
$$

with $\widetilde{G}(\infty)=\infty$, and $\widetilde{G}^{-1}$ is the inverse of $\widetilde{G}$. 
Proof. Let the right side of (3.63) is $z(t), \varepsilon>0$ is an arbitrary small constant, and $J_{\varepsilon}(t)$ is defined as in Theorem 3.7. Then, $u(t) \leq z^{1 / p}(t), t \in \mathbb{T}_{0}$, and similar to the process of (3.53)(3.58), we obtain

$$
\begin{aligned}
z^{(p-q) / p}(t) \leq & J_{\varepsilon}(t)+\int_{t_{0}}^{t} f\left(\tau_{1}(s)\right) z^{(p-q) / p}(s) \Delta s \\
& +\int_{t_{0}}^{t}\left[f\left(\tau_{1}(s)\right) \int_{t_{0}}^{s} g\left(\tau_{1}(\xi)\right) \omega\left(z^{1 / p}(\xi)\right) \Delta \xi\right] \Delta s .
\end{aligned}
$$

Fix $T \in \mathbb{T}_{0}$, and let $t \in\left[t_{0}, T\right] \cap \mathbb{T}$. Considering $J_{\varepsilon}(t)$ is nondecreasing, we deduce that

$$
\begin{aligned}
z^{(p-q) / p}(t) \leq & J_{\varepsilon}(T)+\int_{t_{0}}^{t} f\left(\tau_{1}(s)\right) z^{(p-q) / p}(s) \Delta s \\
& +\int_{t_{0}}^{t}\left[f\left(\tau_{1}(s)\right) \int_{t_{0}}^{s} g\left(\tau_{1}(\xi)\right) \omega\left(z^{1 / p}(\xi)\right) \Delta \xi\right] \Delta s .
\end{aligned}
$$

Denote the right side of (3.66) by $v(t)$. Then, $z^{(p-q) / p}(t) \leq v(t), t \in\left[t_{0}, T\right] \cap \mathbb{T}$, and

$$
\begin{aligned}
v^{\Delta}(t) & =f\left(\tau_{1}(t)\right) z^{(p-q) / p}(t)+f\left(\tau_{1}(t)\right) \int_{t_{0}}^{t} g\left(\tau_{1}(\xi)\right) \omega\left(z^{1 / p}(\xi)\right) \Delta \xi \\
& \leq f\left(\tau_{1}(t)\right) v(t)+f\left(\tau_{1}(t)\right) \int_{t_{0}}^{t} g\left(\tau_{1}(\xi)\right) \omega\left(v^{1 /(p-q)}(\xi)\right) \Delta \xi .
\end{aligned}
$$

Denote $m(t)=v(t)+\int_{t_{0}}^{t} g\left(\tau_{1}(\xi)\right) \omega\left(v^{1 /(p-q)}(\xi)\right) \Delta \xi$. Then, $v(t) \leq m(t)$, and

$$
v^{\Delta}(t) \leq f\left(\tau_{1}(t)\right) m(t)
$$

Furthermore,

$$
\begin{aligned}
m^{\Delta}(t) & =v^{\Delta}(t)+g\left(\tau_{1}(t)\right) \omega\left(v^{1 /(p-q)}(t)\right) \leq f\left(\tau_{1}(t)\right) m(t)+g\left(\tau_{1}(t)\right) \omega\left(m^{1 /(p-q)}(t)\right) \\
& \leq\left[f\left(\tau_{1}(t)\right)+g\left(\tau_{1}(t)\right)\right]\left[m(t)+\omega\left(m^{1 /(p-q)}(t)\right)\right]
\end{aligned}
$$

that is,

$$
\frac{m^{\Delta}(t)}{m(t)+\omega\left(m^{1 /(p-q)}(t)\right)} \leq f\left(\tau_{1}(t)\right)+g\left(\tau_{1}(t)\right) .
$$

On the other hand, similar to (3.21)-(3.23), we have

$$
[\widetilde{G}(m(t))]^{\Delta} \leq \frac{m^{\Delta}(t)}{m(t)+\omega\left(m^{1 /(p-q)}(t)\right)} .
$$


So combining (3.71) and (3.72), we deduce

$$
[\tilde{G}(m(t))]^{\Delta} \leq f\left(\tau_{1}(t)\right)+g\left(\tau_{1}(t)\right)
$$

Considering $m\left(t_{0}\right)=z\left(t_{0}\right)=J_{\varepsilon}(T)$, an integration for (3.73) from $t_{0}$ to $t$ yields

$$
\tilde{G}(m(t)) \leq \tilde{G}\left(J_{\varepsilon}(T)\right)+\int_{t_{0}}^{t}\left[f\left(\tau_{1}(\xi)\right)+g\left(\tau_{1}(\xi)\right)\right] \Delta \xi .
$$

Since $\widetilde{G}$ is increasing, then

$$
m(t) \leq \tilde{G}^{-1}\left\{\tilde{G}\left(J_{\varepsilon}(T)\right)+\int_{t_{0}}^{t}\left[f\left(\tau_{1}(\xi)\right)+g\left(\tau_{1}(\xi)\right)\right] \Delta \xi\right\}
$$

Combining (3.69) and (3.75), we obtain

$$
v^{\Delta}(t) \leq f\left(\tau_{1}(t)\right) \tilde{G}^{-1}\left\{\tilde{G}\left(J_{\mathcal{\varepsilon}}(T)\right)+\int_{t_{0}}^{t}\left[f\left(\tau_{1}(\xi)\right)+g\left(\tau_{1}(\xi)\right)\right] \Delta \xi\right\} .
$$

Setting $t=T$ in (3.76), since $T$ is selected from $\mathbb{T}_{0}$ arbitrarily, then in fact (3.76) holds for all $t \in \mathbb{T}_{0}$, that is,

$$
v^{\Delta}(t) \leq f\left(\tau_{1}(t)\right) \tilde{G}^{-1}\left\{\tilde{G}\left(J_{\varepsilon}(t)\right)+\int_{t_{0}}^{t}\left[f\left(\tau_{1}(\xi)\right)+g\left(\tau_{1}(\xi)\right)\right] \Delta \xi\right\}, \quad \forall t \in \mathbb{T}_{0} .
$$

Considering $v\left(t_{0}\right)=J_{\varepsilon}(T)$, an integration for (3.77) from $t_{0}$ to $t$ yields

$$
v(t) \leq J_{\varepsilon}(T)+\int_{t_{0}}^{t} f\left(\tau_{1}(s)\right) \tilde{G}^{-1}\left\{\tilde{G}\left(J_{\varepsilon}(s)\right)+\int_{t_{0}}^{s}\left[f\left(\tau_{1}(\xi)\right)+g\left(\tau_{1}(\xi)\right)\right] \Delta \xi\right\} \Delta s,
$$

which implies

$$
\begin{aligned}
u(t) \leq\{ & J_{\varepsilon}(T)+\int_{t_{0}}^{t} f\left(\tau_{1}(s)\right) \tilde{G}^{-1} \\
& \left.\times\left\{\tilde{G}\left(J_{\varepsilon}(s)\right)+\int_{t_{0}}^{s}\left[f\left(\tau_{1}(\xi)\right)+g\left(\tau_{1}(\xi)\right)\right] \Delta \xi\right\} \Delta s\right\}^{1 /(p-q)}, \quad t \in\left[t_{0}, T\right] \cap \mathbb{T} .
\end{aligned}
$$

Setting $t=T$ in the above inequality, considering $T$ is selected from $\mathbb{T}_{0}$ arbitrarily, after replacing $T$ with $t$ and letting $\varepsilon \rightarrow 0$, we get the desired result. So the proof is complete. 
Remark 3.10. If we take $\mathbb{T}=\mathbb{R}, t_{0}=0, p=2, q=1, \tau_{1}(t)=\tau_{2}(t)=t$, then Theorem 3.9 reduces to [33, Theorem 3(a7)]. If we take $\mathbb{T}=\mathbb{Z}, t_{0}=0, p=2, q=1, \tau_{1}(t)=\tau_{2}(t)=t$, then Theorem 3.9 reduces to [33, Theorem $6(\mathrm{~b} 7)]$.

Following in a similar manner as the proof of Theorem 3.7 and 3.9, then we present two more theorems as follows, the special cases of which $\left(p=2, q=1, t_{0}=0, \tau_{1}(t)=\right.$ $\left.\tau_{2}(t)=t\right)$ unify the continuous [33, Theorems $\left.1(\mathrm{a} 3), 3(\mathrm{a} 8)\right]$ and discrete inequalities [33, Theorems 4(b3), 6(b8)].

Theorem 3.11. Suppose $u, f, g, h, p, q, C, \alpha, \phi, \tau_{i}, i=1,2$ are defined as in Theorem 3.9. If for $t \in \mathbb{T}_{0}$, $u(t)$ satisfies the following inequality:

$$
\begin{aligned}
u^{p}(t) \leq C^{p /(p-q)}+\frac{p}{p-q} \int_{t_{0}}^{t}\left[f\left(\tau_{1}(s)\right) u^{q}\left(\tau_{1}(s)\right)\right. & \\
\quad & \quad \int_{t_{0}}^{s} g\left(\tau_{1}(\xi)\right) u^{p-q}\left(\tau_{1}(\xi) \Delta \xi+h\left(\tau_{2}(s)\right) u^{q}\left(\tau_{2}(s)\right)\right] \Delta s
\end{aligned}
$$

with the initial condition (3.5), then

$$
u(t) \leq\left[J(t) e_{H}\left(t, t_{0}\right)\right]^{1 /(p-q)}, \quad t \in \mathbb{T}_{0}
$$

provided $H \in \mathfrak{R}_{+}$, where $H(t)=f\left(\tau_{1}(t)\right) \int_{t_{0}}^{t} g\left(\tau_{1}(\xi)\right) \Delta \xi$, and $J(t)$ is defined as in Theorem 3.7.

Theorem 3.12. Suppose $u, f, g, h, \omega, p, q, C, \alpha, \phi, \tau_{i}, i=1,2$ are defined as in Theorem 3.9. If for $t \in \mathbb{T}_{0}, u(t)$ satisfies the following inequality:

$$
\begin{aligned}
u^{p}(t) \leq C^{p /(p-q)}+\frac{p}{p-q} \int_{t_{0}}^{t}\left[f\left(\tau_{1}(s)\right) u^{q}\left(\tau_{1}(s)\right)\right. & \\
& \times \int_{t_{0}}^{s} g\left(\tau_{1}(\xi)\right) \omega\left(u\left(\tau_{1}(\xi)\right) \Delta \xi+h\left(\tau_{2}(s)\right) u^{q}\left(\tau_{2}(s)\right)\right] \Delta s
\end{aligned}
$$

with the initial condition (3.5), then

$$
u(t) \leq\left\{G^{-1}\left[G(J(t))+\int_{t_{0}}^{t} f\left(\tau_{1}(s)\right) \int_{t_{0}}^{s} g\left(\tau_{1}(\xi)\right) \Delta \xi \Delta s\right]\right\}^{1 /(p-q)}, \quad t \in \mathbb{T}_{0}, t \in \mathbb{T}_{0}
$$

where $G$ is defined as in Theorem 3.2, and $J(t)$ is defined as in Theorem 3.7.

\section{Some Applications}

In this section, we will present some applications for the results which we have established above and apply them to qualitative and quantitative analysis of solutions of certain delay dynamic equations on time scales. 
Example 4.1. Consider the following delay dynamic integral equation on time scales:

$$
u^{p}(t)=C+\int_{t_{0}}^{t} F(\tau(s), u(\tau(s))) \Delta s, \quad t \in \mathbb{T}_{0}
$$

with the initial condition

$$
\begin{gathered}
u(t)=\phi(t), \quad t \in\left[\alpha, t_{0}\right] \cap \mathbb{T}, \\
|\phi(\tau(t))| \leq|C|^{1 / p}, \quad \forall t \in \mathbb{T}_{0}, \tau(t) \leq t_{0},
\end{gathered}
$$

where $u \in C_{\mathrm{rd}}\left(\mathbb{T}_{0}, \mathbb{R}\right), \tau \in\left(\mathbb{T}_{0}, \mathbb{T}\right)$ with $\tau(t) \leq t,-\infty<\alpha=\inf \left\{\tau(t), t \in \mathbb{T}_{0}\right\} \leq t_{0}, \phi \in$ $C_{\mathrm{rd}}\left(\left[\alpha, t_{0}\right] \cap \mathbb{T}, \mathbb{R}\right), F \in C_{\mathrm{rd}}(([\alpha, \infty) \cap \mathbb{T}) \times \mathbb{R}, \mathbb{R})$, and $p$ is a constant with $p>1$.

Theorem 4.2. Suppose $u(t)$ is a solution of (4.1)-(4.2), and $|F(t, u)| \leq f(t)|u|^{p}+g(t)|u|^{p-1}$, where $f, g \in C_{r d}\left([\alpha, \infty) \cap \mathbb{T}, \mathbb{R}_{+}\right)$, then

$$
u(t) \leq\left[C+\int_{t_{0}}^{t} g(\tau(s)) \Delta s\right] \exp \left[\int_{t_{0}}^{t} f(\tau(s)) \Delta s\right], \quad t \in \mathbb{T}_{0} .
$$

Proof. In fact, from (4.1) we have

$$
\begin{aligned}
|u(t)|^{p} & \leq|C|+\int_{t_{0}}^{t}|F(\tau(s), u(\tau(s)))| \Delta s \\
& \leq|C|+\int_{t_{0}}^{t}\left[f(\tau(s))|u(\tau(s))|^{p}+g(\tau(s))|u(\tau(s))|^{p-1}\right] \Delta s \\
& =|C|+\int_{t_{0}}^{t}\left[f(\tau(s))|u(\tau(s))|^{p-1} \omega(|u(\tau(s))|)+g(\tau(s))|u(\tau(s))|^{p-1}\right] \Delta s,
\end{aligned}
$$

where $\omega(r)=r$. Then a suitable application of Theorem $3.2\left(\right.$ with $\tau_{1}=\tau_{2}=\tau, q=p-1$ ) to (4.4) yields

$$
u(t) \leq G^{-1}\left[G\left(C+\int_{t_{0}}^{t} g(\tau(s)) \Delta s\right)+\int_{t_{0}}^{t} f(\tau(s)) \Delta s\right], \quad t \in \mathbb{T}_{0}
$$

where $G(x)=\int_{1}^{x}(1 / r) d r=\ln x$, for all $x>0$. Using the expression of $G$ in (4.5), we obtain the desired result, and the proof is complete.

Example 4.3. Consider the following delay dynamic differential equation on time scales

$$
\left(u^{3}(t)\right)^{\Delta}=F\left[\tau(t), u(\tau(t)), \int_{t_{0}}^{t} M(\tau(\xi), u(\tau(\xi))) \Delta \xi\right], \quad t \in \mathbb{T}_{0}
$$


with the initial condition

$$
\begin{gathered}
u\left(t_{0}\right)=u_{0}, \\
u(t)=\phi(t), \quad t \in\left[\alpha, t_{0}\right] \cap \mathbb{T}, \\
|\phi(\tau(t))| \leq\left|u_{0}\right|, \quad \forall t \in \mathbb{T}_{0}, \tau(t) \leq t_{0},
\end{gathered}
$$

where $u, \tau, \alpha, \phi$ are defined as in Example 4.1, $F \in C_{\mathrm{rd}}\left(([\alpha, \infty) \cap \mathbb{T}) \times \mathbb{R}^{2}, \mathbb{R}\right), M \in C_{\mathrm{rd}}(([\alpha, \infty) \cap$ $\mathbb{T}) \times \mathbb{R}, \mathbb{R})$.

Theorem 4.4. Suppose $u(t)$ is a solution of (4.6)-(4.7), and $|F(t, u, v)| \leq f(t)|u|^{3}+$ $f(t)|u||v|,|M(t, u)| \leq g(t)|u|^{2}$, where $f, g \in C_{\mathrm{rd}}\left([\alpha, \infty) \cap \mathbb{T}, \mathbb{R}_{+}\right)$, then

$$
u(t) \leq\left|u_{0}\right| \sqrt{\left[1+\int_{t_{0}}^{t} f(\tau(s)) e_{(f+g)(\tau(\cdot))}\left(s, t_{0}\right) \Delta s\right]}, \quad t \in \mathbb{T}_{0}
$$

provided $(f+g)(\tau(\cdot)) \in \mathfrak{R}_{+}$.

Proof. The equivalent integral form of (4.6)-(4.7) is denoted by

$$
u^{3}(t)=u_{0}^{3}+\int_{t_{0}}^{t} F\left[\tau(s), u(\tau(s)), \int_{t_{0}}^{s} M(\tau(\xi), u(\tau(\xi))) \Delta \xi\right] \Delta s
$$

So,

$$
\begin{aligned}
|u(t)|^{3} & \leq\left|u_{0}\right|^{3}+\int_{t_{0}}^{t}\left|F\left[\tau(s), u(\tau(s)), \int_{t_{0}}^{s} M(\tau(\xi), u(\tau(\xi))) \Delta \xi\right]\right| \Delta s \\
& \leq\left|u_{0}\right|^{3}+\int_{t_{0}}^{t}\left[f(\tau(s))|u(\tau(s))|^{3}+f(\tau(s))|u(\tau(s))|\left|\int_{t_{0}}^{s} M(\tau(\xi), u(\tau(\xi))) \Delta \xi\right|\right] \Delta s \\
& \leq\left|u_{0}^{2}\right|^{3 / 2}+\int_{t_{0}}^{t}\left[f(\tau(s))|u(\tau(s))|^{3}+f(\tau(s))|u(\tau(s))| \int_{t_{0}}^{s} g(\tau(\xi))|u(\tau(\xi))|^{2} \Delta \xi\right] \Delta s .
\end{aligned}
$$

Then a suitable application of Theorem 3.7 (with $p=3, q=1, \tau_{1}=\tau_{2}=\tau, h(t) \equiv 0$ ) to (4.10) yields the desired inequality (4.8), and the proof is complete.

Theorem 4.5. If under the conditions of Theorem 4.4, for $t \in \mathbb{T}_{0}, \int_{t_{0}}^{t} f\left(\tau_{1}(s)\right) e_{(f+g)\left(\tau_{1}(.)\right)}\left(s, t_{0}\right) \Delta s \leq$ $L$, where $L>0$ is a constant, then the trivial solution of (4.6)-(4.7) is uniformly stable.

Theorem 4.6. Assume $\left|F\left(t, u_{1}, v_{1}\right)-F\left(t, u_{2}, v_{2}\right)\right| \leq f(t)\left|u_{1}^{3}-u_{2}^{3}\right|+f(t) \sqrt{\left|u_{1}^{3}-u_{2}^{3}\right| \mid v_{1}-}$ $v_{2}|| M,\left(t, u_{1}\right)-M\left(t, u_{2}\right)|\leq g(t)| \sqrt{\left|u_{1}^{3}-u_{2}^{3}\right|}$, where $f, g$ are defined as in Theorem 4.4, then (4.6)(4.7), have at most one solution. 
Proof. Suppose $u_{1}(t), u_{2}(t)$ are two solutions of (4.6)-(4.7). By (4.9) we have

$$
\begin{aligned}
\left|u_{1}^{3}(t)-u_{2}^{3}(t)\right| \leq \int_{t_{0}}^{t} \mid F\left[\tau(s), u_{1}(\tau(s)), \int_{t_{0}}^{s} M\left(\tau(\xi), u_{1}(\tau(\xi))\right) \Delta \xi\right] \\
\quad-F\left[\tau(s), u_{2}(\tau(s)), \int_{t_{0}}^{s} M\left(\tau(\xi), u_{2}(\tau(\xi))\right) \Delta \xi\right] \mid \Delta s \\
\leq \int_{t_{0}}^{t}\left[f(\tau(s))\left|u_{1}^{3}(\tau(s))-u_{2}^{3}(\tau(s))\right|+f(\tau(s)) \sqrt{\left|u_{1}^{3}(\tau(s))-u_{2}^{3}(\tau(s))\right|}\right. \\
\left.\quad \times \int_{t_{0}}^{s}\left[M\left(\tau(\xi), u_{1}(\tau(\xi))\right)-M\left(\tau(\xi), u_{2}(\tau(\xi))\right)\right] \Delta \xi\right] \Delta s \\
\leq \int_{t_{0}}^{t}\left[f(\tau(s))\left|u_{1}^{3}(\tau(s))-u_{2}^{3}(\tau(s))\right|+f(\tau(s)) \sqrt{\left|u_{1}^{3}(\tau(s))-u_{2}^{3}(\tau(s))\right|}\right. \\
\quad \times \int_{t_{0}}^{s} g(\tau(\xi)) \sqrt{\left.\left|u_{1}^{3}(\tau(\xi))-u_{2}^{3}(\tau(\xi))\right| \Delta \xi\right] \Delta s .}
\end{aligned}
$$

Treat $\left|u_{1}^{3}(t)-u_{2}^{3}(t)\right|$ as one variable, and applying Theorem 3.7 to (4.11) (with $p=1, q=$ $\left.1 / 2, \tau_{1}=\tau_{2}=\tau, h(t) \equiv 0\right)$ yields $\left|u_{1}^{3}-u_{2}^{3}\right| \leq 0$, which implies $u_{1}(t) \equiv u_{2}(t)$, and the proof is complete.

\section{Acknowledgments}

This work is partially supported by National Natural Science Foundation of China (11171178 and 11101248), Natural Science Foundation of Shandong Province (ZR2009AM011, ZR2010AQ026, and ZR2010AZ003) (China), and Specialized Research Fund for the Doctoral Program of Higher Education (20103705 110003)(China). The authors thank the referees very much for their careful comments and valuable suggestions on this paper.

\section{References}

[1] T. H. Gronwall, "Note on the derivatives with respect to a parameter of the solutions of a system of differential equations," Annals of Mathematics Second Series, vol. 20, no. 4, pp. 292-296, 1919.

[2] R. Bellman, "The stability of solutions of linear differential equations," Duke Mathematical Journal, vol. 10, pp. 643-647, 1943.

[3] B. G. Pachpatte, Inequalities for Differential and Integral Equations, vol. 197 of Mathematics in Science and Engineering, Academic Press, New York, NY, USA, 1998.

[4] F. Jiang and F. Meng, "Explicit bounds on some new nonlinear integral inequalities with delay," Journal of Computational and Applied Mathematics, vol. 205, no. 1, pp. 479-486, 2007.

[5] O. Lipovan, "Integral inequalities for retarded Volterra equations," Journal of Mathematical Analysis and Applications, vol. 322, no. 1, pp. 349-358, 2006.

[6] E. Yang, "On some nonlinear integral and discrete inequalities related to Ou-Iang's inequality," Acta Mathematica Sinica New Series, vol. 14, no. 3, pp. 353-360, 1998.

[7] Q.-H. Ma, "Estimates on some power nonlinear Volterra-Fredholm type discrete inequalities and their applications," Journal of Computational and Applied Mathematics, vol. 233, no. 9, pp. 2170-2180, 2010. 
[8] R. P. Agarwal, S. Deng, and W. Zhang, "Generalization of a retarded Gronwall-like inequality and its applications," Applied Mathematics and Computation, vol. 165, no. 3, pp. 599-612, 2005.

[9] B. G. Pachpatte, "Inequalities applicable in the theory of finite difference equations," Journal of Mathematical Analysis and Applications, vol. 222, no. 2, pp. 438-459, 1998.

[10] R. A. C. Ferreira and D. F. M. Torres, "Generalized retarded integral inequalities," Applied Mathematics Letters, vol. 22, no. 6, pp. 876-881, 2009.

[11] L. Ou Yang, "The boundedness of solutions of linear differential equations $y$ " $A(t) y=0$," Shuxue Jinzhan, vol. 3, pp. 409-415, 1957.

[12] W.-S. Cheung, "Some new nonlinear inequalities and applications to boundary value problems," Nonlinear Analysis: Theory, Methods \& Applications, vol. 64, no. 9, pp. 2112-2128, 2006.

[13] W.-S. Cheung and J. Ren, "Discrete non-linear inequalities and applications to boundary value problems," Journal of Mathematical Analysis and Applications, vol. 319, no. 2, pp. 708-724, 2006.

[14] S. Hilger, "Analysis on measure chains-a unified approach to continuous and discrete calculus," Results in Mathematics, vol. 18, no. 1-2, pp. 18-56, 1990.

[15] M. Bohner, L. Erbe, and A. Peterson, “Oscillation for nonlinear second order dynamic equations on a time scale," Journal of Mathematical Analysis and Applications, vol. 301, no. 2, pp. 491-507, 2005.

[16] M. J. Bohner, R. A. C. Ferreira, and D. F. M. Torres, "Integral inequalities and their applications to the calculus of variations on time scales," Mathematical Inequalities $\mathcal{E}$ Applications, vol. 13, no. 3, pp. 511-522, 2010.

[17] R. Agarwal, M. Bohner, D. O'Regan, and A. Peterson, "Dynamic equations on time scales: a survey," Journal of Computational and Applied Mathematics, vol. 141, no. 1-2, pp. 1-26, 2002.

[18] F.-H. Wong, C.-C. Yeh, and W.-C. Lian, "An extension of Jensen's inequality on time scales," Advances in Dynamical Systems and Applications, vol. 1, no. 1, pp. 113-120, 2006.

[19] H. M. Srivastava, K.-L. Tseng, S.-J. Tseng, and J.-C. Lo, "Some generalizations of Maroni's inequality on time scales," Mathematical Inequalities E Applications, vol. 14, no. 2, pp. 469-480, 2011.

[20] M. Bohner and T. Matthews, "The Grüss inequality on time scales," Communications in Mathematical Analysis, vol. 3, no. 1, pp. 1-8, 2007.

[21] Q.-A. Ngô, "Some mean value theorems for integrals on time scales," Applied Mathematics and Computation, vol. 213, no. 2, pp. 322-328, 2009.

[22] W. Liu and Q.-A. Ngô, "Some Iyengar-type inequalities on time scales for functions whose second derivatives are bounded," Applied Mathematics and Computation, vol. 216, no. 11, pp. 3244-3251, 2010.

[23] W. Liu and Q.-A. Ngô, "A generalization of Ostrowski inequality on time scales for points," Applied Mathematics and Computation, vol. 203, no. 2, pp. 754-760, 2008.

[24] M. Z. Sarikaya, "On weighted Iyengar type inequalities on time scales," Applied Mathematics Letters, vol. 22, no. 9, pp. 1340-1344, 2009.

[25] W. N. Li, "Some delay integral inequalities on time scales," Computers \& Mathematics with Applications, vol. 59, no. 6, pp. 1929-1936, 2010.

[26] Q.-H. Ma and J. Pečarić, "The bounds on the solutions of certain two-dimensional delay dynamic systems on time scales," Computers \& Mathematics with Applications, vol. 61, no. 8, pp. 2158-2163, 2011.

[27] W. N. Li, "Some new dynamic inequalities on time scales," Journal of Mathematical Analysis and Applications, vol. 319, no. 2, pp. 802-814, 2006.

[28] R. Agarwal, M. Bohner, and A. Peterson, "Inequalities on time scales: a survey," Mathematical Inequalities E Applications, vol. 4, no. 4, pp. 535-557, 2001.

[29] M. Bohner and A. Peterson, Dynamic Equations on Time Scales: An Introduction with Applications, Birkhäuser, Boston, Mass, USA, 2001.

[30] W. N. Li, "Some Pachpatte type inequalities on time scales," Computers \& Mathematics with Applications, vol. 57, no. 2, pp. 275-282, 2009.

[31] Y. G. Sun, “On retarded integral inequalities and their applications," Journal of Mathematical Analysis and Applications, vol. 301, no. 2, pp. 265-275, 2005.

[32] O. Lipovan, "A retarded integral inequality and its applications," Journal of Mathematical Analysis and Applications, vol. 285, no. 2, pp. 436-443, 2003.

[33] B. G. Pachpatte, "On some new inequalities related to certain inequalities in the theory of differential equations," Journal of Mathematical Analysis and Applications, vol. 189, no. 1, pp. 128-144, 1995.

[34] L. Li, F. Meng, and P. Ju, "Some new integral inequalities and their applications in studying the stability of nonlinear integro-differential equations with time delay," Journal of Mathematical Analysis and Applications, vol. 377, no. 2, pp. 853-862, 2011.

[35] B. G. Pachpatte, "On a new inequality suggested by the study of certain epidemic models," Journal of Mathematical Analysis and Applications, vol. 195, no. 3, pp. 638-644, 1995. 


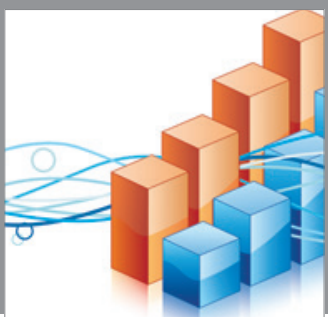

Advances in

Operations Research

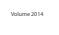

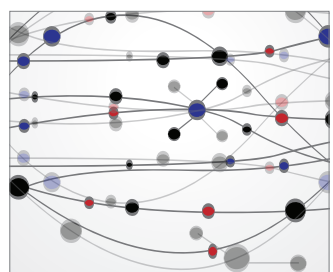

\section{The Scientific} World Journal
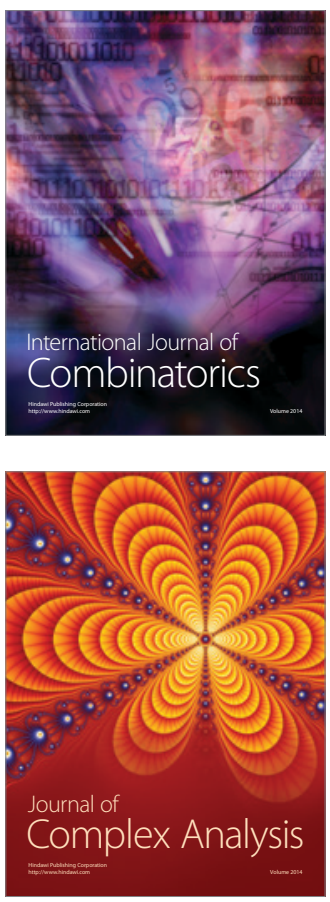

International Journal of

Mathematics and

Mathematical

Sciences
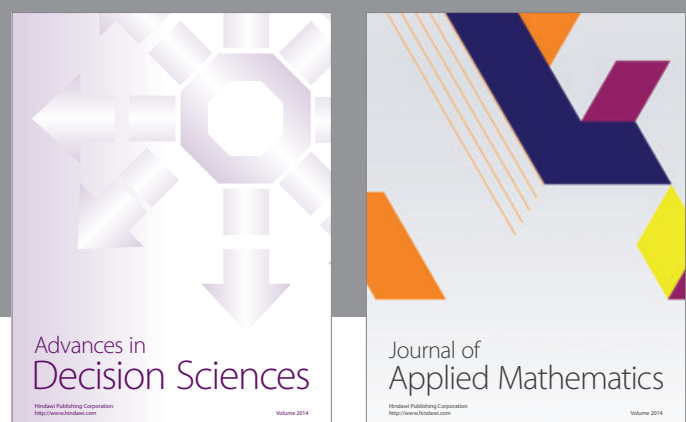

Journal of

Applied Mathematics
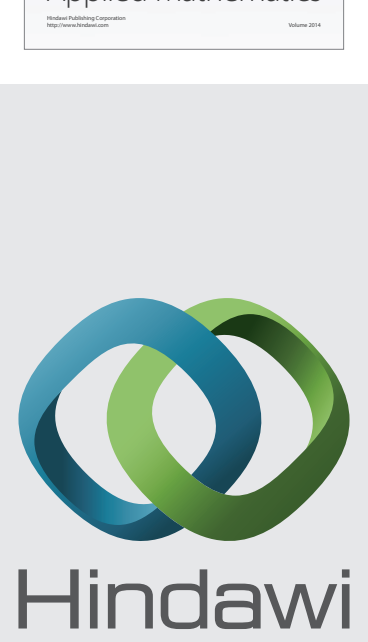

Submit your manuscripts at http://www.hindawi.com
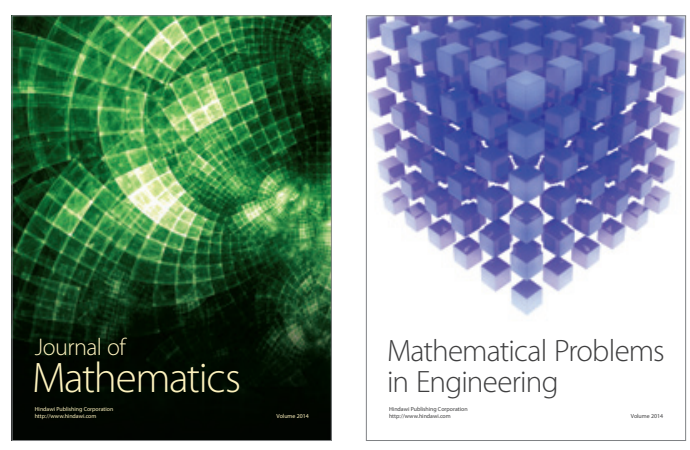

Mathematical Problems in Engineering
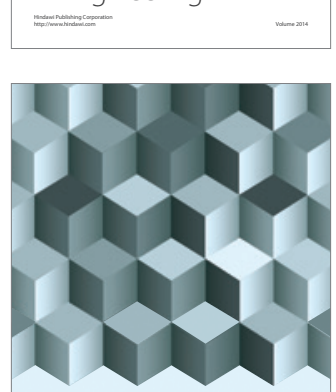

Journal of

Function Spaces
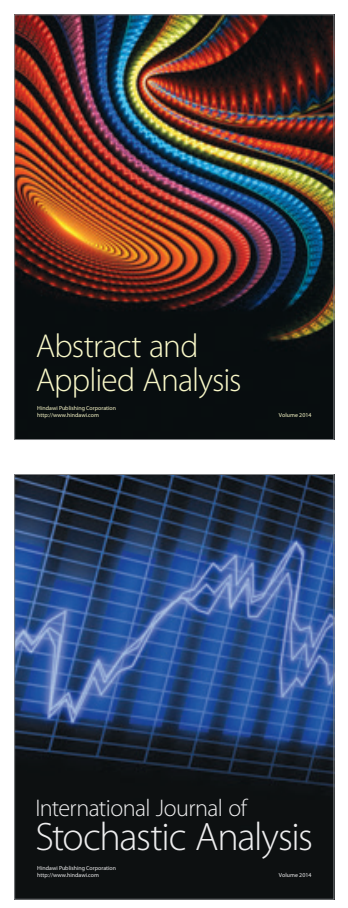

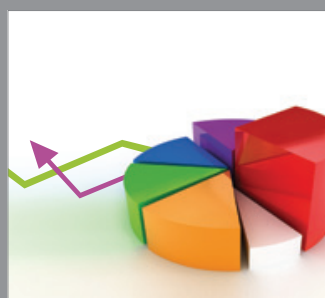

ournal of

Probability and Statistics

Promensencen
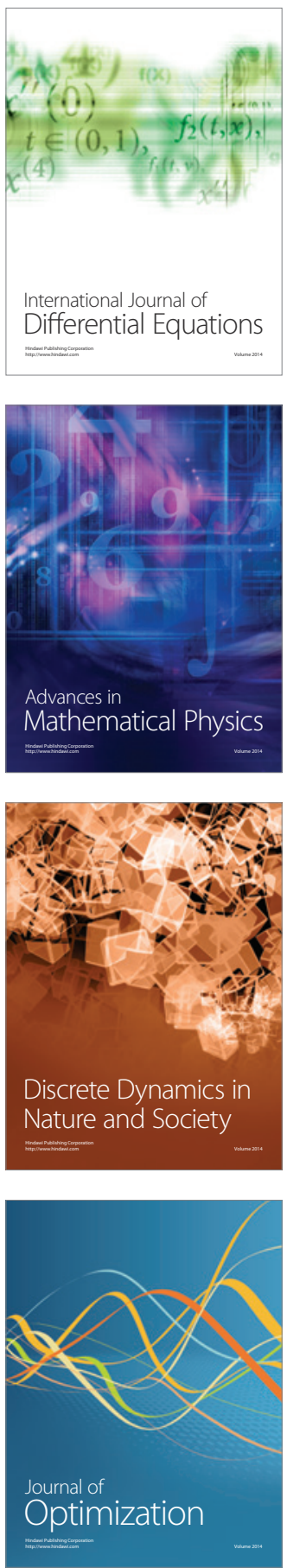